PROCEEDINGS OF THE

AMERICAN MATHEMATICAL SOCIETY

Volume 130, Number 1, Pages 193-203

S 0002-9939(01)05072-9

Article electronically published on July 31, 2001

\title{
LADDER SYSTEMS ON TREES
}

\author{
ZORAN SPASOJEVIĆ \\ (Communicated by Andreas R. Blass)
}

\begin{abstract}
We formulate the notion of uniformization of colorings of ladder systems on subsets of trees. We prove that Suslin trees have this property and also Aronszajn trees in the presence of Martin's Axiom. As an application we show that if a tree has this property, then every countable discrete family of subsets of the tree can be separated by a family of pairwise disjoint open sets. Such trees are then normal and hence countably paracompact. As a dual result for special Aronszajn trees we prove that the weak diamond, $\Phi_{\omega}$, implies that no special Aronszajn tree can be countably paracompact.
\end{abstract}

\section{$\S 1$. INTRODUCTION}

Topological properties of trees have been extensively studied, particularly of trees with specific structure such as Suslin, almost Suslin and special Aronszajn trees. It was proved in [2] that Suslin trees are normal and that a tree is almost Suslin if and only if it is collectionwise Hausdorff. Starting from Martin's Axion (MA), Fleissner [4] proved that every special Aronszajn tree is normal and Hanazawa [6] pointed out that normality implies countable paracompactness in trees. In addition, Fleissner [5] further studied topological properties of trees and proved the equivalence of collection-wise normality to other properties of trees. In $\S 2$ we formulate a combinatorial property for $\omega_{1}$-trees which, among other properties, incorporates in it normality and hence countable paracompactness. We then prove that Suslin trees have this property and also Aronszajn trees if MA $+\neg \mathrm{CH}$ holds.

On the other hand starting from $\nabla_{S}\left(\forall\right.$ stationary $\left.S \subseteq \omega_{1}\right)$, Watson [8] established that no special Aronszajn tree can be countably paracompact. In $\S 3$ we recall the notion of a weak diamond $\Phi_{n}(2 \leq n \leq \omega)$ studied in [7] and prove that under $\Phi_{\omega}$ the property of $\S 2$ fails for special Aronszajn trees by proving that $\Phi_{\omega}$ implies that no special Aronszajn tree can be countably paracompact. Thus, in light of the fact that $\nabla_{S}\left(\forall\right.$ stationary $\left.S \subseteq \omega_{1}\right)$ is strictly stronger than $\diamond$ which in turn is strictly stronger than $\Phi_{\omega}$, we also improve the result of Watson stated above.

We now formulate some basic notions and establish the notation which will be used in this paper. If $\alpha$ and $\beta$ are ordinals, then $\alpha^{\beta}=\{f: f: \beta \rightarrow \alpha\}$ and $\alpha^{<\beta}=$ $\bigcup_{\nu<\beta} \alpha^{\nu}$. For a set $A,[A]^{<\omega}$ denotes the collection of all finite subsets of $A$ and $[A]^{\kappa}$ the collection of all subsets of $A$ of size $\kappa$. A tree, $\mathbb{T}=\left\langle T, \leq_{T}\right\rangle$, is a partial order in the strict sense such that for each $t \in T$ the set $\hat{t}=\left\{s \in T: s<_{T} t\right\}$ is well ordered by $\leq_{T}$. If $t \in T$, the height of $t$ in $\mathbb{T}, \operatorname{ht}(t, \mathbb{T})$, is the ordinal $\alpha$ which is the order

Received by the editors June 5, 1996 and, in revised form, May 18, 1998.

2000 Mathematics Subject Classification. Primary 03E05.

(C)2001 American Mathematical Society 
type of $\hat{t}$. Let $T^{\prime}=\{t \in T: \operatorname{ht}(t, \mathbb{T})$ is a limit ordinal $\}$. For each ordinal $\alpha$, the $\alpha$ th level of $\mathbb{T}, \operatorname{Lev}_{\alpha}(\mathbb{T})$, is the set $\{t \in T: \operatorname{ht}(t, \mathbb{T})=\alpha\}$ and $T_{\alpha}=\bigcup_{\nu<\alpha} \operatorname{Lev}_{\nu}(\mathbb{T})$. The height of $\mathbb{T}, \operatorname{ht}(\mathbb{T})$, is the least ordinal $\alpha$ such that $\operatorname{Lev}_{\alpha}(\mathbb{T})=\emptyset$. If $A \subseteq T$ and $C \subseteq \omega_{1}$, then $A\lceil C=\{t \in A: \operatorname{ht}(t, \mathbb{T}) \in C\}$. And $A \subseteq T$ is stationary iff $\{\operatorname{ht}(t, \mathbb{T}): t \in A\}$ is stationary in $\omega_{1}$, i.e. has nonempty intersection with every closed unbounded subset of $\omega_{1}$. An $\alpha$-branch of $\mathbb{T}$ is a set $B \subseteq T$ such that $B$ is well ordered by $\leq_{T}$ in order type $\alpha$, and $\forall \beta<\alpha\left(B \cap \operatorname{Lev}_{\beta}(\mathbb{T}) \neq \emptyset\right)$. An anti-chain in $\mathbb{T}$ is a set $A \subseteq T$ such that $\forall s, t \in A\left(s \neq t \rightarrow s \mathbb{Z}_{T} t \wedge t \mathbb{L}_{T} s\right)$. More generally, an anti-chain in a partial order is a set whose elements are pairwise incompatible (for trees this means incomparable). Compatibility of $p$ and $q$ in a partial order will be denoted by $p \sim q$ and their incompatibility by $p \nsim q$.

We will only consider well pruned trees. A well pruned tree is a tree $\mathbb{T}=\left\langle T, \leq_{T}\right\rangle$ such that

(1) $\left|\operatorname{Lev}_{0}(\mathbb{T})\right|=1$,

(2) $\forall \alpha<\beta<\operatorname{ht}(\mathbb{T}) \forall t \in \operatorname{Lev}_{\alpha}(\mathbb{T}) \exists s_{1}, s_{2} \in \operatorname{Lev}_{\beta}(\mathbb{T})\left(s_{1} \neq s_{2} \wedge t \leq_{T} s_{1}, s_{2}\right)$,

(3) $\forall \alpha<\operatorname{ht}(\mathbb{T}) \forall s, t \in \operatorname{Lev}_{\alpha}(\mathbb{T})($ limit $\alpha \rightarrow(s=t \leftrightarrow \hat{s}=\hat{t}))$.

From this point on all trees are well pruned. An $\omega_{1}$-tree is a well pruned tree $\mathbb{T}=\left\langle T, \leq_{T}\right\rangle$ such that ht $(\mathbb{T})=|T|=\omega_{1}$ and $\forall \alpha<\operatorname{ht}(\mathbb{T})\left(\left|\operatorname{Lev}_{\alpha}(\mathbb{T})\right| \leq \omega\right)$. With the exception of a few observations below, all the results in this paper deal with trees which do not have any $\omega_{1}$-branches. Such trees are given specific names. Any $\omega_{1}$-tree without $\omega_{1}$-branch is called an Aronszajn tree. An Aronszajn tree is special if it is a union of countably many anti-chains. It is a consequence of $\mathrm{MA}+\neg \mathrm{CH}$ that every Aronszajn tree is special. An $\omega_{1}$-tree is Suslin if it does not contain any uncountable anti-chains; it is almost Suslin if it does not contain any stationary anti-chains. Almost Suslin trees were introduced in [2] where it was shown that if there is a Suslin tree, then there is an almost Suslin tree which is not Suslin. Thus, by countable completeness of the nonstationary ideal on $\omega_{1}$, special Aronszajn trees are not almost Suslin. The existence of Suslin trees is denied by MA $+\neg \mathrm{CH}$.

We also consider trees as topological spaces. For a tree $\mathbb{T}=\left\langle T, \leq_{T}\right\rangle$, a basis for a topology on $\mathbb{T}$ is the following collection of sets:

$$
\{\hat{t}: t \in T\} \cup\left\{\left\{u \in T: s<_{T} u<_{T} t\right\}: s, t \in T\right\} .
$$

For $s, t \in T$, with $s \leq_{T} t$, we let $(s, t)_{T}=\left\{u \in T: s<_{T} u<_{T} t\right\}$ and such sets will be called intervals in $\mathbb{T}$. We also use the notation $[s, t)_{T},(s, t]_{T},[s, t]_{T}$ to specify whether or not the interval includes the end points.

All topological spaces in this paper are Hausdorff. Let $X$ be one such space. $X$ is countably paracompact if every countable open cover of $X$ has a locally finite open refinement. All other topological notions appearing in this paper are standard and can be found in any text book on the subject. We point out that in a tree with topology as above any discrete set of points is also closed. Thus any reference below to a discrete set implies closed and discrete.

Then an $\omega_{1}$-tree is normal, $\ldots$ if it is normal, ... as a topological space with the topology described above. Suslin trees are normal and it was proved in [2] that a tree is almost Suslin iff it is collection-wise Hausdorff. Other topological properties of trees were mentioned in the earlier part of this section.

\section{$\S 2$. LADDER SYSTEMS}

Devlin and Shelah [1] formulated the notion of uniformization of colorings of ladder systems on $\omega_{1}$. We consider an analogous notion on $\omega_{1}$-trees. 
Definition 1. Let $\mathbb{T}=\left\langle T, \leq_{T}\right\rangle$ be an $\omega_{1}$-tree and $A \subseteq T^{\prime}$. A ladder system on $A$ is a collection $\tilde{\eta}_{A}=\left\langle\eta_{t}: t \in A\right\rangle$ such that each $\eta_{t}$ is a $<_{T}$-increasing sequence with limit $t$. $\tilde{\eta}_{A}$ is a full ladder system if for each $t \in A$ there is $r<_{T} t$ such that $\eta_{t}=[r, t)_{T}$. A coloring of $\tilde{\eta}_{A}$ is a collection $\tilde{c}_{\tilde{\eta}_{A}}=\left\langle c_{t}: t \in A\right\rangle$ such that $c_{t}: \eta_{t} \rightarrow \omega$ for each $t \in A$. $\tilde{c}_{\tilde{\eta}_{A}}$ is a nice coloring if it satisfies the following two conditions:

(1) $\forall t \in A \exists s<_{T} t \forall u \in A \exists v<_{T} u \forall a \in \eta_{t} \backslash \hat{s} \cap \eta_{u} \backslash \hat{v}\left(c_{t}(a)=c_{u}(a)\right)$,

(2) $\forall t \in A \exists \varphi_{t}\left(\varphi_{t}\right.$ is a function $\wedge \varphi_{t}: \omega \rightarrow \eta_{t} \wedge \forall m, n<\omega\left(m<n \rightarrow \varphi_{t}(m)<_{T}\right.$ $\left.\varphi_{t}(n)\right) \wedge \sup _{T}\left\{\varphi_{t}(n): n<\omega\right\}=t \wedge \forall n<\omega\left(\mid\left\{c_{t}(a): \varphi_{t}(n) \leq_{T} a<_{T}\right.\right.$ $\left.\left.\left.\varphi_{t}(n+1)\right\} \mid=1\right)\right)$.

A function $f: T \rightarrow \omega$ uniformizes the coloring $\tilde{c}_{\tilde{\eta}_{A}}$ if $\forall t \in A \exists s<_{T} t \forall a \in \eta_{t} \backslash$ $\hat{s}\left(c_{t}(a)=f(a)\right)$.

We point out that condition (1) is a nontrivial assertion only in the case when ht $(t, \mathbb{T})$ is a limit of limit ordinals and there is a $B \subseteq A \cap \hat{t}$, which is $\leq_{T}$-unbounded in $\hat{t}$, such that $\forall u \in B\left(\sup _{T}\left(\eta_{t} \cap \eta_{u}\right)=u\right)$. Otherwise, it is easy to see that there is always an $s<_{T} t$ such that $\forall u \in A \exists v<_{T} u\left(\eta_{t} \backslash \hat{s} \cap \eta_{u} \backslash \hat{v}=\emptyset\right)$ so that condition (1) holds vacuously. It is also easy to see that if a coloring does not satisfy condition (1), then it cannot be uniformized. Thus, condition (1) is a necessary condition for a uniformizing function to exist. Condition (2) above states that for each $t \in A$ and $s \in T$, with $\varphi_{t}(0) \leq_{T} s<_{T} t, c_{t}$ has only finitely many color changes on the interval $\left[\varphi_{t}(0), s\right)_{T}$. It is a nontrivial assertion only in the case when $\operatorname{ht}(t, \mathbb{T})$ is a limit of limit ordinals. Otherwise, there is always a function $\varphi_{t}$ with the desired properties.

The next example justifies the inclusion of condition (2) in the above definition. In fact, the example shows that it is also a necessary requirement on colorings of ladder systems on subsets of Aronszajn trees for uniformizing functions to exist under MA $+\neg \mathrm{CH}$. It is easy to see in condition (2) that for each $n<\omega$ if we require that domain of $\varphi_{t}$ is $\omega \cdot n$ instead of $\omega$ and retain the other requirements, then we get an equivalent condition. The next example shows that we cannot require that domain $\varphi_{t}$ is $\omega \cdot \omega$ for trees which are not almost Suslin such as, for example, special Aronszajn trees. It is an open question if there is an analogous example for Suslin or almost Suslin trees. Since condition (2) seems to play an essential role in the proof of Theorem 2 below, this might be evidence that there are similar examples for such trees as well.

Let $\mathbb{T}=\left\langle T, \leq_{T}\right\rangle$ be an $\omega_{1}$-tree which is not almost Suslin and let $A$ be a stationary anti-chain in $T$. Since $\mathbb{T}$ is well pruned we may assume that the height of each element of $A$ is a limit of limit ordinals. For each $t \in A$ fix an $s_{t}<_{T} t$ and let $\eta_{t}=\left[s_{t}, t\right)_{T}$. Then $\tilde{\eta}_{A}=\left\langle\eta_{t}: t \in A\right\rangle$ is a full ladder system on $A$. For each $t \in A$ let $\varphi_{t}: \omega \cdot \omega \rightarrow \eta_{t}$ be such that $\varphi_{t}(0)=s_{t}, \zeta<\xi<\omega \cdot \omega \rightarrow \varphi_{t}(\zeta)<_{T} \varphi_{t}(\xi)$, and $\sup _{T}\left\{\varphi_{t}(\xi): \xi<\omega \cdot \omega\right\}=t$. Let $B \in\left[\omega^{\omega}\right]^{\omega_{1}}$ and let $\left\{B_{t}: t \in A\right\}$ be a partition of $B$ with $B_{t}=\left\{f_{t}^{i}: i<\omega\right\}$ for each $t \in A$ where $i \neq j \vee t \neq s \rightarrow f_{t}^{i} \neq f_{s}^{j}$. We now define coloring $\tilde{c}_{\tilde{n}_{A}}=\left\langle c_{t}: t \in A\right\rangle$. Fix $t \in A$. For each $i, n<\omega$ and $s \in\left[\varphi_{t}(\omega \cdot n+i), \varphi_{t}(\omega \cdot n+i+1)\right)_{T}$ let $c_{t}(s)=f_{t}^{n}(i)$. This defines $\tilde{c}_{\tilde{\eta}_{A}}$ and it has the property that

$$
\forall i, n<\omega\left(\left|\left\{c_{t}(a): \varphi_{t}(\omega \cdot n+i) \leq_{T} a<_{T} \varphi_{t}(\omega \cdot n+i+1)\right\}\right|=1\right) .
$$

We show that this coloring cannot be uniformized. By way of contradiction assume $f: T \rightarrow \omega$ is a uniformizing function. For each $t \in A$ let $n_{t}<\omega$ be such that $f$ and $c_{t}$ agree on $\left[\varphi_{t}\left(\omega \cdot n_{t}\right), t\right)_{T}$. Since $A$ is stationary and the nonstationary ideal $\mathcal{I}$ 
on $\omega_{1}$ is countably complete we may assume that $\forall t \in A\left(n_{t}=n\right)$ for some fixed $n$. Now by Fodor's Lemma (i.e. Pressing Down Lemma), there is a stationary $A^{\prime} \subseteq A$ such that

$$
\forall t, s \in A^{\prime}\left(\operatorname{ht}\left(\varphi_{t}(\omega \cdot n), \mathbb{T}\right)=\operatorname{ht}\left(\varphi_{s}(\omega \cdot n), \mathbb{T}\right)\right) .
$$

Using countable completeness of $\mathcal{I}$ again, since levels of $\mathbb{T}$ are countable we may, in fact, assume that

$$
\forall t, s \in A^{\prime}\left(\varphi_{t}(\omega \cdot n)=\varphi_{s}(\omega \cdot n)\right) .
$$

Using Fodor's Lemma and countable completeness of $\mathcal{I}$ one more time we get a stationary $A^{\prime \prime} \subseteq A^{\prime}$ such that

$$
\forall t, s \in A^{\prime \prime}\left(\varphi_{t}(\omega \cdot(n+1))=\varphi_{s}(\omega \cdot(n+1))\right) .
$$

Then

$$
\forall t, s \in A^{\prime \prime}\left(\left[\varphi_{t}(\omega \cdot n), \varphi_{t}(\omega \cdot(n+1))\right)_{T}=\left[\varphi_{s}(\omega \cdot n), \varphi_{s}(\omega \cdot(n+1))\right)_{T}\right) .
$$

Now $f_{t}^{n} \neq f_{s}^{n}$ whenever $t, s \in A^{\prime \prime}$ with $t \neq s$ so that whenever $t \neq s$ in $A^{\prime \prime}$ there is an

$$
r \in\left[\varphi_{t}(\omega \cdot n), \varphi_{t}(\omega \cdot(n+1))\right)_{T}
$$

such that $c_{t}(r) \neq c_{s}(r)$. But then either $f(r) \neq c_{t}(r)$ or $f(r) \neq c_{s}(r)$ which contradicts the assumption that $f$ is a uniformizing function for the coloring $\tilde{c}_{\tilde{\eta}_{A}}$. This shows that the requirement that $\operatorname{domain}\left(\varphi_{t}\right)=\omega$ in Definition 1(2) is an optimal requirement for a coloring to have a uniformizing function under $\mathrm{MA}+\neg \mathrm{CH}$ in the case of special Aronszajn trees.

In the following observations, with the exception of (c), $\mathbb{T}$ may have $\omega_{1}$-branches.

(a) If $\mathbb{T}$ is an $\omega_{1}$-tree and $A \subseteq T^{\prime}$ is countable, then any nice coloring of a ladder system on $A$ can be uniformized.

(b) If $\mathbb{T}$ is an $\omega_{1}$-tree and $A \subseteq T^{\prime}$ is nonstationary, then any nice coloring of a ladder system on $A$ can be uniformized.

(c) If $\mathbb{T}$ is almost Suslin and $A \subseteq T^{\prime}$ is discrete, then any coloring of a ladder system on $A$ can be uniformized.

(d) If $\mathrm{MA}+\neg \mathrm{CH}$ holds, $\mathbb{T}$ is an $\omega_{1}$-tree, $A \subseteq T^{\prime}, \tilde{\eta}_{A}=\left\langle\eta_{t}: t \in A\right\rangle$ is a ladder system on $A$ such that each $\eta_{t}$ has order type $\omega$, and $\tilde{c}_{\tilde{\eta}_{A}}$ is any coloring of $\tilde{\eta}_{A}$ (hence nice), then there is a uniformizing function.

To prove (a) enumerate $A$ in order type $\omega$ and proceed inductively using the fact that the coloring is nice. For (b) let $C \subseteq \omega_{1}$ be closed unbounded such that $T \uparrow C$ is disjoint from $A$. Now proceed inductively by using (a) above to define a uniformizing function on the elements of $T$ whose heights lie between consecutive points of $C$. For (c) use the fact that almost Suslin trees are collection-wise Hausdorff. The proof of Theorem 5.2 of [1] can easily be modified to yield (d).

The next theorem shows that normal almost Suslin trees have the uniformization property. But first two lemmas.

Lemma 2. Let $\mathbb{T}=\left\langle T, \leq_{T}\right\rangle$ be an almost Suslin tree and $B \subseteq T$ a discrete set. Then $B$ is not stationary.

Proof. By way of contradiction assume $B$ is stationary. Since $B$ is discrete and $\mathbb{T}$ is almost Suslin, hence collection-wise Hausdorff, for each $t \in B$ there is a $p_{t}<_{T} t$ such that if $t \neq s$ in $B$, then $\left(p_{t}, t\right]_{T} \cap\left(p_{s}, s\right]_{T}=\emptyset$. By Fodor's Lemma, there is a stationary $B^{\prime} \subseteq B$ and an $\alpha<\omega_{1}$ such that $\forall t \in B^{\prime}\left(\operatorname{ht}\left(p_{t}, \mathbb{T}\right)=\alpha\right)$. Since $\operatorname{Lev}_{\alpha}(\mathbb{T})$ 
is countable, by countable completeness of the nonstationary ideal on $\omega_{1}$, there is a $p \in \operatorname{Lev}_{\alpha}(\mathbb{T})$ and a stationary $B^{\prime \prime} \subseteq B^{\prime}$ such that $\forall t \in B^{\prime \prime}\left(p_{t}=p\right)$. For each $t \in B^{\prime \prime}$ let $r_{t} \in(p, t]_{T} \cap \operatorname{Lev}_{\alpha+1}(\mathbb{T})$. Since $(p, s]_{T} \cap(p, t]_{T}=\emptyset$ whenever $s \neq t$ in $B^{\prime \prime}$ it follows that $\forall s, t \in B^{\prime \prime}\left(s \neq t \rightarrow r_{s} \neq r_{t}\right)$. But $\operatorname{Lev}_{\alpha+1}(\mathbb{T})$ is countable, so $B^{\prime \prime}$ is also countable, hence it cannot be stationary, a contradiction.

Lemma 3. Let $\mathbb{T}=\left\langle T, \leq_{T}\right\rangle$ be a normal almost Suslin tree and $B \subseteq T$ discrete. Then there is a discrete family $\left\{U_{b}: b \in B\right\}$ of closed and open sets and a club $C \subseteq \omega_{1}$ such that $\forall b \in B\left(b \in U_{b} \subseteq T\right)$ and $T \uparrow C \cap \bigcup_{b \in B} U_{b}=\emptyset$.

Proof. By the previous lemma, $B$ is not stationary, so let $C \subseteq \omega_{1}$ be a club such that $B \cap T \uparrow C=\emptyset$. Without loss of generality we may assume that $0 \in C$ and that each $\alpha \in C \backslash\{0\}$ is a limit ordinal. Since both $B$ and $T \uparrow C$ are closed, by normality of $\mathbb{T}$, let $U \subseteq T$ be open such that $B \subseteq U$ and $T \uparrow C \cap \bar{U}=\emptyset$. Let $\left\{\alpha_{\xi}: \xi<\omega_{1}\right\}$ be the increasing enumeration of $C$ and for $\xi<\omega_{1}$ let $\left\{b_{i}^{\xi}: i<\omega\right\}$ and $\left\{x_{i}^{\xi}: i<\omega\right\}$ be enumerations of $B \cap\left(T_{\alpha_{\xi+1}} \backslash T_{\alpha_{\xi}}\right)$ and $\bar{U} \cap\left(T_{\alpha_{\xi+1}} \backslash T_{\alpha_{\xi}}\right) \backslash B$ respectively. For each $\xi<\omega_{1}$ we shall construct, by induction, collections $\left\{U_{i}^{\xi}: i<\omega\right\}$ and $\left\{V_{i}^{\xi}: i<\omega\right\}$ of sets such that

(1) $\forall i<\omega\left(U_{i}^{\xi}\right.$ and $V_{i}^{\xi}$ are closed and open),

(2) $\forall i<\omega\left(U_{i}^{\xi} \subseteq U \cap\left(T_{\alpha_{\xi+1}} \backslash T_{\alpha_{\xi}}\right) \wedge V_{i}^{\xi} \subseteq\left(T_{\alpha_{\xi+1}} \backslash T_{\alpha_{\xi}}\right)\right)$,

(3) $\forall i<\omega\left(b_{i}^{\xi} \in U_{i}^{\xi} \wedge x_{i}^{\xi} \in V_{i}^{\xi}\right)$,

(4) $\forall i, j<\omega\left(i \neq j \rightarrow U_{i}^{\xi} \cap U_{j}^{\xi}=\emptyset\right)$,

(5) $\forall i<\omega\left(\exists j<\omega\left(V_{i}^{\xi} \cap U_{j}^{\xi} \neq \emptyset\right) \rightarrow V_{i}^{\xi}=U_{j}^{\xi}\right)$.

So, fix $\xi<\omega_{1}$ and $n<\omega$ and suppose $\left\{U_{i}^{\xi}: i<n\right\}$ and $\left\{V_{i}^{\xi}: i<n\right\}$ have been constructed satisfying (1)-(5) above and also

(6) $\bigcup_{i<n}\left(U_{i}^{\xi} \cup V_{i}^{\xi}\right) \cap\left\{b_{i}^{\xi}: i \geq n\right\}=\emptyset$,

(7) $\forall i<n\left(V_{i}^{\xi} \cap \bigcup_{j<n} U_{j}^{\xi}=\emptyset \rightarrow V_{i}^{\xi} \cap B=\emptyset\right)$.

We consider three cases.

Case 1: $\exists i<n\left(x_{n}^{\xi} \in U_{i}^{\xi}\right)$.

Since $U_{i}^{\xi}$ 's and $V_{i}^{\xi}$ 's are closed, so is $\bigcup_{i<n}\left(U_{i}^{\xi} \cup V_{i}^{\xi}\right)$ and is disjoint from $B \backslash$ $\left\{b_{i}^{\xi}: i<n\right\}$. So let $s<_{T} b_{n}^{\xi}$ be such that $\left(s, b_{n}^{\xi}\right]_{T} \subseteq U \cap\left(T_{\alpha_{\xi+1}} \backslash T_{\alpha_{\xi}}\right)$ and $\left(s, b_{n}^{\xi}\right]_{T} \cap\left(\bigcup_{i<n}\left(U_{i}^{\xi} \cup V_{i}^{\xi}\right) \cup\left(B \backslash\left\{b_{n}^{\xi}\right\}\right)\right)=\emptyset$. In this case let $U_{n}^{\xi}=\left(s, b_{n}^{\xi}\right]_{T}$ and $V_{n}^{\xi}=U_{i}^{\xi}$ and note that (1)-(7) above are satisfied.

Case 2: $\exists i<n\left(x_{n}^{\xi} \in V_{i}^{\xi} \wedge \forall j<n\left(V_{i}^{\xi} \cap U_{j}^{\xi}=\emptyset\right)\right)$.

Since $U_{i}^{\xi}$ 's and $V_{i}^{\xi}$ 's are closed, so is $\bigcup_{i<n}\left(U_{i}^{\xi} \cup V_{i}^{\xi}\right)$ and is disjoint from $B \backslash$ $\left\{b_{i}^{\xi}: i<n\right\}$. So let $s<_{T} b_{n}^{\xi}$ be such that $\left(s, b_{n}^{\xi}\right]_{T} \subseteq U \cap\left(T_{\alpha_{\xi+1}} \backslash T_{\alpha_{\xi}}\right)$ and $\left(s, b_{n}^{\xi}\right]_{T} \cap\left(\bigcup_{i<n}\left(U_{i}^{\xi} \cup V_{i}^{\xi}\right) \cup\left(B \backslash\left\{b_{n}^{\xi}\right\}\right)\right)=\emptyset$. In this case let $U_{n}^{\xi}=\left(s, b_{n}^{\xi}\right]_{T}$ and $V_{n}^{\xi}=V_{i}^{\xi}$ and note that (1)-(7) above are satisfied.

Case 3: $x_{n}^{\xi} \notin \bigcup_{i<n}\left(U_{i}^{\xi} \cup V_{i}^{\xi}\right)$.

Using the hypothesis on $U_{i}^{\xi}$ 's and $V_{i}^{\xi}$ 's and the fact that $\mathbb{T}$ is Hausdorff and $B$ is discrete let $s<_{T} b_{n}^{\xi}$ and $r<_{T} x_{n}^{\xi}$ be such that $\left(s, b_{n}^{\xi}\right]_{T} \subseteq U \cap\left(T_{\alpha_{\xi+1}} \backslash T_{\alpha_{\xi}}\right),\left(r, x_{n}^{\xi}\right]_{T} \subseteq$ $T_{\alpha_{\xi+1}} \backslash T_{\alpha_{\xi}},\left(s, b_{n}^{\xi}\right]_{T} \cap\left(r, x_{n}^{\xi}\right]_{T}=\emptyset,\left(s, b_{n}^{\xi}\right]_{T} \cap\left(\bigcup_{i<n}\left(U_{i}^{\xi} \cup V_{i}^{\xi}\right) \cup\left(B \backslash\left\{b_{n}^{\xi}\right\}\right)\right)=\emptyset$, $\left(r, x_{n}^{\xi}\right]_{T} \cap \bigcup_{i<n}\left(U_{i}^{\xi} \cup V_{i}^{\xi} \cup B\right)=\emptyset$. In this case let $U_{n}^{\xi}=\left(s, b_{n}^{\xi}\right]_{T}$ and $V_{n}^{\xi}=\left(r, x_{n}^{\xi}\right]_{T}$ and note that (1)-(7) above are satisfied.

It is now easy to see that $\left\{U_{i}^{\xi}: i<\omega\right\}$ and $\left\{V_{i}^{\xi}: i<\omega\right\}$ satisfy (1)-(5) above. For each $b \in B, b=b_{n}^{\xi}$ for some $\xi<\omega_{1}$ and $n<\omega$. Let $U_{b}=U_{n}^{\xi}$. Then each $U_{b}$ 
is closed and open, $b \in U_{b}$ and $T \uparrow C \cap \bigcup_{b \in B} U_{b}=\emptyset$ since $\forall b \in B\left(U_{b} \subseteq U\right)$ and $\bar{U} \cap T \uparrow C=\emptyset$. In addition $\left\{U_{b}: b \in B\right\}$ is a pairwise disjoint family. In fact it is a discrete family. To see this let $t \in T$. If $t \in \bar{U}$, then $t=b_{n}^{\xi}$ or $t=x_{n}^{\xi}$ for some $\xi<\omega_{1}$ and $n<\omega$. Then $U_{n}^{\xi}$ or $V_{n}^{\xi}$ witnesses that $t$ has an open neighborhood which has a nonempty intersection with at most one element in $\left\{U_{b}: b \in B\right\}$. And if $t \notin \bar{U}$, then $T \backslash \bar{U}$ is an open neighborhood of $t$ which is disjoint from $\bigcup_{b \in B} U_{b}$. This proves the lemma.

Theorem 4. Let $\mathbb{T}$ be a normal almost Suslin tree and suppose that $A \subseteq T^{\prime}$ is closed in T. Then every nice coloring of a full ladder system on $A$ can be uniformized.

Proof. Let $\tilde{\eta}_{A}=\left\langle\eta_{t}: t \in A\right\rangle$ be a full ladder system on $A$ and $\tilde{c}_{\tilde{\eta}_{A}}=\left\langle c_{t}: t \in A\right\rangle$ a nice coloring of $\tilde{\eta}_{A}$. Let $B=\left\{t \in A: c_{t}\right.$ is not eventually constant $\}$. Then $B$ is discrete in $T$. To see this, by way of contradiction, assume $B$ has an accumulation point. Since $A$ is closed this point is in $A$. But $\tilde{c}_{\tilde{\eta}_{A}}$ is nice so for all $t$ in some neighborhood of this accumulation point, $c_{t}$ is eventually constant, and this is a contradiction by the definition of $B$. Then by Lemma $2, B$ is not stationary in $T$.

Let $C \subseteq \omega_{1}$ be closed unbounded such that $T\lceil C$ is disjoint from $B$. We may assume that $0 \in C$ and that each $\alpha \in C \backslash\{0\}$ is a limit ordinal. Let $\left\{U_{b}: b \in B\right\}$ be as in Lemma 3 and $V=T \backslash \bigcup_{b \in B} U_{b}$. Then, since $\left\{U_{b}: b \in B\right\}$ is a discrete family of closed and open sets, $V$ is also closed and open in $T$ and $T \uparrow C \subseteq V$. Now for each $t \in A \cap V, c_{t}$ is eventually constant. So for $i<\omega$ let $A_{i}=\{t \in$ $A \cap V: c_{t}$ is eventually equal to $\left.i\right\}$. Since $A \cap V$ is closed in $T$ and $\tilde{c}_{\tilde{\eta}_{A}}$ is nice it follows that each $A_{i}$ is closed in $T, A_{i} \cap A_{j}=\emptyset$ for $i \neq j$, and that $\left\{A_{i}: i<\omega\right\}$ is a discrete family. Since normality implies countable collection-wise normality there are pairwise disjoint open sets $V_{i}$ such that $A_{i} \subseteq V_{i} \subseteq V$ for $i<\omega$. We may assume that $\bigcup_{i<\omega} V_{i}=V$. Now we define a uniformizing $f: T \rightarrow \omega$. If $t \in A_{i}$, then for $s \in \eta_{t} \cap V_{i}$ let $f(s)=i$. This defines $f$ on $V \cap \bigcup_{t \in A} \eta_{t}$. We define now $f$ on $(T \backslash V) \cap \bigcup_{t \in A} \eta_{t}$. Let $\left\{\alpha_{\nu}: \nu<\omega_{1}\right\}$ be the monotone enumeration of $C$. We proceed by induction on $\nu$. Since $A \cap\left(T_{\alpha_{\nu+1}} \backslash\left(T_{\alpha_{\nu}} \cup V\right)\right)$ is countable, we use observation (a) to define $f$ on $\left(T_{\alpha_{\nu+1}} \backslash\left(T_{\alpha_{\nu}} \cup V\right)\right) \cap \bigcup_{t \in A} \eta_{t}$. Note that $T_{\alpha_{\nu+1}} \backslash\left(T_{\alpha_{\nu}} \cup V\right)$ is closed and open in $T$ so $A \cap\left(T_{\alpha_{\nu+1}} \backslash\left(T_{\alpha_{\nu}} \cup V\right)\right)$ is closed, hence contains all of its accumulation points so there is no danger of conflict with the previously defined values of $f$. This defines $f$ on each $\eta_{t}(t \in A)$. Now extend $f$ arbitrarily to cover all of $T$. Clearly $f$ uniformizes $\tilde{c}_{\tilde{\eta}_{A}}$ and the theorem is proved.

An immediate corollary is that if $\mathbb{T}$ is Suslin and $A \subseteq T^{\prime}$ is closed, then every nice coloring of a full ladder system on $A$ can be uniformized.

Our next task is to show that if MA $+\neg \mathrm{CH}$ holds, $\mathbb{T}$ is Aronszajn and $A \subseteq T^{\prime}$, then every nice coloring of a full ladder system on $A$ can be uniformized. Let $\tilde{\eta}_{A}=\left\langle\eta_{t}: t \in A\right\rangle$ be a ladder system on $A$ and $\tilde{c}_{\tilde{\eta}_{A}}=\left\langle c_{t}: t \in A\right\rangle$ a nice coloring of $\tilde{\eta}_{A}$. Let $t \in A$ and let $s<_{T} t$ be as in Definition 1(1) and $\varphi_{t}$ as in Definition 1(2). For the rest of this section fix a $p_{t} \in T$ such that $s, \varphi_{t}(0) \leq_{T} p_{t}<_{T} t$. If $\mathrm{ht}(t, \mathbb{T})$ is not a limit of limit ordinals (it is always a limit ordinal since $t \in A \subseteq T^{\prime}$ ), then also require that $p_{t}$ succeeds the immediate predecessor of $t$ in $T^{\prime}$. For each $x \in[A \times T]^{<\omega}$ also let $x^{0}=\{t: \exists s((t, s) \in x)\}$. We now define a partial order intended to uniformize $\tilde{c}_{\tilde{\eta}_{A}}$. 
Definition 5. Let $\mathbb{T}, A, \tilde{\eta}_{A}, \tilde{c}_{\tilde{\eta}_{A}}, p_{t}(t \in A)$ be as above. Let

$$
\begin{aligned}
\mathbb{P}_{A \tilde{\eta} \tilde{c}}= & \left\{(x, g): x \in[A \times T]<\omega \wedge \forall(t, s) \in x\left(p_{t} \leq_{T} s<_{T} t\right) \wedge\right. \\
& \left.g \text { is a function } \wedge g: \bigcup_{(t, s) \in x} \eta_{t} \backslash \hat{s} \rightarrow \omega \wedge \forall(t, s) \in x \forall a \in \eta_{t} \backslash \hat{s}\left(g(a)=c_{t}(a)\right)\right\}
\end{aligned}
$$

where $\left(x_{2}, g_{2}\right) \leq\left(x_{1}, g_{1}\right)$ iff $x_{2} \supseteq x_{1}$ and $g_{2} \supseteq g_{1}$.

Lemma 6. Let $\mathbb{T}=\left\langle T, \leq_{T}\right\rangle$ be a special Aronszajn tree, $A \subseteq T^{\prime}, \tilde{\eta}_{A}=\left\langle\eta_{t}: t \in A\right\rangle$ a full ladder system on $A$, and $\tilde{c}_{\tilde{\eta}_{A}}=\left\langle c_{t}: t \in A\right\rangle$ a nice coloring of $\tilde{\eta}_{A}$. Then $\mathbb{P}_{A \tilde{\eta} \tilde{c}}$ has the ccc.

Proof. By way of contradiction, let $\left\{\left(x_{\alpha}, g_{\alpha}\right): \alpha<\omega_{1}\right\}$ be an uncountable antichain in $\mathbb{P}_{A \tilde{\eta} \tilde{c}}$. As $\tilde{\eta}_{A}$ is a full ladder system, $\forall \alpha<\omega_{1} \forall(t, s) \in x_{\alpha}\left(\eta_{t} \backslash \hat{s}=[s, t)_{T}\right)$. By the $\Delta$-system lemma we may assume that $\left\{x_{\alpha}^{0}: \alpha<\omega_{1}\right\}$ forms a $\Delta$-system with root $r$ and after another thinning process we may assume $r=\emptyset$. Since $\forall \alpha, \beta<\omega_{1}\left(\alpha \neq \beta \rightarrow\left(x_{\alpha}, g_{\alpha}\right) \not\left(x_{\beta}, g_{\beta}\right)\right)$ we have

$(\diamond) \quad \forall \alpha, \beta<\omega_{1}\left(\alpha \neq \beta \rightarrow \exists(t, s) \in x_{\alpha} \exists\left(t^{\prime}, s^{\prime}\right) \in x_{\beta}\right.$

$$
\left.\exists a \in[s, t)_{T} \cap\left[s^{\prime}, t^{\prime}\right)_{T}\left(g_{\alpha}(a) \neq g_{\beta}(a)\right)\right) .
$$

For all $\alpha<\omega_{1}$ and each $(t, s) \in x_{\alpha}$ there is $r_{t}$, with $s \leq_{T} r_{t} \leq_{T} t$, such that $\bigcup_{(t, s) \in x_{\alpha}}[s, t)_{T}=\bigcup_{t \in x_{\alpha}^{0}}\left[r_{t}, t\right)_{T}$ and $\forall t, t^{\prime} \in x_{\alpha}^{0}\left(t \neq t^{\prime} \rightarrow\left[r_{t}, t\right)_{T} \cap\left[r_{t^{\prime}}, t^{\prime}\right)_{T}=\emptyset\right)$. For each $\alpha<\omega_{1}$ let $y_{\alpha}=\left\{\left(t, r_{t}\right): t \in x_{\alpha}^{0} \wedge r_{t}<_{T} t\right\}$. Then, since $\operatorname{dom}\left(g_{\alpha}\right)=$ $\bigcup_{(t, s) \in x_{\alpha}}[s, t)_{T}=\bigcup_{(t, r) \in y_{\alpha}}[r, t)_{T}$ for each $\alpha<\omega_{1}$, we have $\forall \alpha<\omega_{1}\left(\left(y_{\alpha}, g_{\alpha}\right) \in\right.$

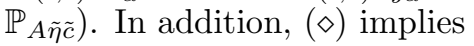

(о) $\forall \alpha, \beta<\omega_{1}\left(\alpha \neq \beta \rightarrow \exists(t, s) \in y_{\alpha} \exists\left(t^{\prime}, s^{\prime}\right) \in y_{\beta}\right.$

$$
\left.\exists a \in[s, t)_{T} \cap\left[s^{\prime}, t^{\prime}\right)_{T}\left(g_{\alpha}(a) \neq g_{\beta}(a)\right)\right) .
$$

Thus $\left\{\left(y_{\alpha}, g_{\alpha}\right): \alpha<\omega_{1}\right\}$ is an uncountable anti-chain in $\mathbb{P}_{A \tilde{\eta} \tilde{c}}$.

From now on we work with the uncountable anti-chain $\left\{\left(y_{\alpha}, g_{\alpha}\right): \alpha<\omega_{1}\right\}$ toward a contradiction. We may assume that $\forall \alpha<\omega_{1}\left(\left|y_{\alpha}\right|=n\right)$ for some $n<\omega$. So for each $\alpha<\omega_{1}$ let $y_{\alpha}=\left\{\left(t_{i}^{\alpha}, r_{i}^{\alpha}\right): i<n\right\}$ and let $\varphi_{t_{i}^{\alpha}}$ be as in Definition 1(2). For each $\alpha<\omega_{1}$ and $i<n$ we define $\psi_{i}^{\alpha}: \omega \rightarrow\left[r_{i}^{\alpha}, t_{i}^{\alpha}\right)_{T}$ as follows. Let $l_{i}^{\alpha}$ be the least such that $r_{i}^{\alpha}<_{T} \varphi_{t_{i}^{\alpha}}\left(l_{i}^{\alpha}\right)$. Let $\psi_{i}^{\alpha}(0)=r_{i}^{\alpha}$ and $\psi_{i}^{\alpha}(j)=\varphi_{t_{i}^{\alpha}}\left(l_{i}^{\alpha}+j-1\right)$ for $0<j<\omega$. By another thinning process we may assume

$$
\forall \beta<\omega_{1}\left(\sup \left\{\operatorname{ht}\left(t_{i}^{\alpha}, \mathbb{T}\right): i<n \wedge \alpha<\beta\right\}<\min \left\{\operatorname{ht}\left(\psi_{i}^{\beta}\left(m_{\beta}\right), \mathbb{T}\right): i<n\right\}\right)
$$

for some $m_{\beta}<\omega$. We may further assume that $\forall \alpha<\omega_{1}\left(m_{\alpha}=m\right)$ for some $m<\omega$. Now $\mathbb{T}=\left\langle T, \leq_{T}\right\rangle$ is a special Aronszajn tree, so let $T=\bigcup_{l<\omega} A_{l}$ where each $A_{l}(l<\omega)$ is an anti-chain in $\mathbb{T}$. By a further thinning process we may assume

$$
\forall i<n \forall k \leq m \exists l<\omega\left(\left\{\psi_{i}^{\alpha}(k): \alpha<\omega_{1}\right\} \subseteq A_{l}\right) .
$$

And by another thinning process we may assume

$$
\begin{aligned}
& \forall \alpha, \beta<\omega_{1} \forall i<n \forall k<m \\
& \forall a \in\left[\psi_{i}^{\alpha}(k), \psi_{i}^{\alpha}(k+1)\right)_{T} \forall b \in\left[\psi_{i}^{\beta}(k), \psi_{i}^{\beta}(k+1)\right)_{T}\left(c_{t_{i}^{\alpha}}(a)=c_{t_{i}^{\beta}}(b)\right) .
\end{aligned}
$$

This in turn implies

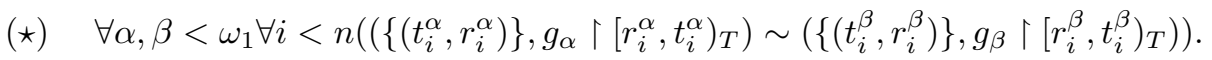


Let $\mathcal{U}$ be a uniform ultrafilter on $\omega_{1}$. Then (o) implies that for each $\alpha<\omega_{1}$ there are $i(\alpha), j(\alpha)<n$ such that

$$
B_{\alpha}=\left\{\beta: \alpha<\beta<\omega_{1} \wedge \exists a \in\left[r_{i(\alpha)}^{\alpha}, t_{i(\alpha)}^{\alpha}\right)_{T} \cap\left[r_{j(\alpha)}^{\beta}, t_{j(\alpha)}^{\beta}\right)_{T}\left(g_{\alpha}(a) \neq g_{\beta}(a)\right)\right\} \in \mathcal{U} .
$$

Furthermore, there must be $i, j<n$ such that

$$
B=\{\alpha: i(\alpha)=i \wedge j(\alpha)=j\} \in \mathcal{U} \text {. }
$$

Note that by $(\star)$ we have $i \neq j$. Let $\alpha \in B$ and note that $B \cap B_{\alpha} \in \mathcal{U}$. Let $\beta \in B \cap B_{\alpha}$ and note that $B \cap B_{\alpha} \cap B_{\beta} \in \mathcal{U}$. Let $\gamma \in B \cap B_{\alpha} \cap B_{\beta}$ and note that $\alpha<\beta<\gamma$. We also have that

$$
\begin{aligned}
& \exists a \in\left[r_{i}^{\alpha}, t_{i}^{\alpha}\right)_{T} \cap\left[r_{j}^{\beta}, t_{j}^{\beta}\right)_{T}\left(g_{\alpha}(a) \neq g_{\beta}(a)\right), \\
& \exists a \in\left[r_{i}^{\alpha}, t_{i}^{\alpha}\right)_{T} \cap\left[r_{j}^{\gamma}, t_{j}^{\gamma}\right)_{T}\left(g_{\alpha}(a) \neq g_{\gamma}(a)\right), \\
& \exists a \in\left[r_{i}^{\beta}, t_{i}^{\beta}\right)_{T} \cap\left[r_{j}^{\gamma}, t_{j}^{\gamma}\right)_{T}\left(g_{\beta}(a) \neq g_{\gamma}(a)\right) .
\end{aligned}
$$

But this implies

$$
\begin{aligned}
& {\left[r_{i}^{\alpha}, t_{i}^{\alpha}\right)_{T} \cap\left[r_{j}^{\beta}, t_{j}^{\beta}\right)_{T} \neq \emptyset,} \\
& {\left[r_{i}^{\alpha}, t_{i}^{\alpha}\right)_{T} \cap\left[r_{j}^{\gamma}, t_{j}^{\gamma}\right)_{T} \neq \emptyset,} \\
& {\left[r_{i}^{\beta}, t_{i}^{\beta}\right)_{T} \cap\left[r_{j}^{\gamma}, t_{j}^{\gamma}\right)_{T} \neq \emptyset .}
\end{aligned}
$$

This in turn implies $\left[r_{i}^{\beta}, t_{i}^{\beta}\right)_{T} \cap\left[r_{j}^{\beta}, t_{j}^{\beta}\right)_{T} \neq \emptyset$ which contradicts the earlier assumption that $\left[r_{i}^{\beta}, t_{i}^{\beta}\right)_{T} \cap\left[r_{j}^{\beta}, t_{j}^{\beta}\right)_{T}=\emptyset$. Therefore $\left\{\left(y_{\alpha}, g_{\alpha}\right): \alpha<\omega_{1}\right\}$ cannot be an uncountable anti-chain in $\mathbb{P}_{A \tilde{\eta} \tilde{c}}$ and hence $\left\{\left(x_{\alpha}, g_{\alpha}\right): \alpha<\omega_{1}\right\}$ cannot be an uncountable anti-chain in $\mathbb{P}_{A \tilde{\eta} \tilde{c}}$. This proves the lemma.

Lemma 7. Let $\mathbb{T}, A, \tilde{\eta}_{A}, \tilde{c}_{\tilde{\eta}_{A}}$ be as in the previous lemma. For each $t \in A$, the sets $D_{t}=\left\{(x, g) \in \mathbb{P}_{A \tilde{\eta} \tilde{c}}: t \in x^{0}\right\}$ are dense in $\mathbb{P}_{A \tilde{\eta} \tilde{c}}$.

Proof. Fix $t \in A$ and let $(x, g) \in \mathbb{P}_{A \tilde{y} \tilde{c}}$. If $t \in x^{0}$, there is nothing to show. So suppose $t \notin x^{0}$. Using Definition 1(1) we choose $s \in T$, with $p_{t} \leq_{T} s<_{T} t$, such that

$$
\forall(u, v) \in x \forall a \in[s, t)_{T} \cap[v, u)_{T}\left(c_{t}(a)=c_{u}(a)\right) .
$$

Let $y=x \cup\{(t, s)\}$, $\operatorname{dom}(h)=\operatorname{dom}(g) \cup[s, t)_{T}, h \uparrow \operatorname{dom}(g)=g$, and $\forall a \in$ $[s, t)_{T}\left(h(a)=c_{t}(a)\right)$. Clearly $(y, h) \in \mathbb{P}_{A \tilde{\eta} \tilde{c}},(y, h) \in D_{t}$, and $(y, h) \leq(x, g)$ so that $D_{t}$ is a dense subset of $\mathbb{P}_{A \tilde{\eta} \tilde{c}}$.

Theorem 8. Assume MA $+\neg \mathrm{CH}$. If $\mathbb{T}=\left\langle T, \leq_{T}\right\rangle$ is an Aronszajn tree, $A \subseteq T^{\prime}$, $\tilde{\eta}_{A}$ is a full ladder system on $A$, and $\tilde{c}_{\tilde{\eta}_{A}}$ is a nice coloring of $\tilde{\eta}_{A}$, then $\tilde{c}_{\tilde{\eta}_{A}}$ can be uniformized.

Proof. Since MA $+\neg \mathrm{CH}$ holds, $\mathbb{T}$ is a special Aronszajn tree. Let $\tilde{\eta}_{A}=\left\langle\eta_{t}: t \in A\right\rangle$ and $\tilde{c}_{\tilde{\eta}_{A}}=\left\langle c_{t}: t \in A\right\rangle$ be as in the statement of the theorem. Let $\mathbb{P}_{A \tilde{\eta} \tilde{c}}$ and $D_{t}(t \in$ $A)$ be as above. Then $\mathbb{P}_{A \tilde{\eta} \tilde{c}}$ has the $c c c$ and $D_{t}(t \in A)$ is dense. By MA $+\neg \mathrm{CH}$ let $G \subseteq \mathbb{P}_{A \tilde{\eta} \tilde{c}}$ be a filter which meets each $D_{t}(t \in A)$. Let $\tilde{f}=\bigcup\{g: \exists x((x, g) \in G)\}$. Clearly, for each $t \in A$ there is $s<_{T} t$ such that $\forall a \in[s, t)_{T}\left(\tilde{f}(a)=c_{t}(a)\right)$. But $\tilde{f}$ may not be a total function on $T$. To rectify this, define $f: T \rightarrow \omega$ by letting $f \uparrow \operatorname{dom}(\tilde{f})=\tilde{f}$ and $f(a)=0$ for all $a \in T \backslash \operatorname{dom}(\tilde{f})$. Then $f$ is a uniformizing function for the coloring $\tilde{c}_{\tilde{\eta}_{A}}$ and the theorem is proved. 
In Theorem 4 normality played a crucial role in proving that the uniformization property for $\mathbb{T}$ holds. And since $\mathrm{MA}+\neg \mathrm{CH}$ implies that all Aronszajn trees are normal, normality is also present in trees as in Theorem 8. This suggests that normality and the uniformization property in trees are closely related. In fact, normality is implied by the uniformization property, as the next result shows. In fact, together with Theorem 4, the next result shows that for almost Suslin trees, normality is equivalent to the existence of uniformizing functions for nice colorings of full ladder systems on closed sets. We state it in a somewhat more general form.

Theorem 9. Suppose $\mathbb{T}$ is an $\omega_{1}$-tree such that for each closed $A \subseteq T^{\prime}$, each nice coloring of a full ladder system on $A$ can be uniformized. Then any countable discrete family of subsets of $\mathbb{T}$ can be separated by pairwise disjoint open sets.

Proof. Let $\left\{A_{n}: n<\omega\right\}$ be a countable discrete family of subsets of $T$. Then $\left\{\bar{A}_{n}: n<\omega\right\}$ is also discrete and let $A=\bigcup_{n<\omega} \bar{A}_{n} \cap T^{\prime}$. We define a full ladder system, $\tilde{\eta}_{A}=\left\langle\eta_{t}: t \in A\right\rangle$, on $A$ and a nice coloring, $\tilde{c}_{\tilde{\eta}_{A}}=\left\langle c_{t}: t \in A\right\rangle$, of $\tilde{\eta}_{A}$. Let $t \in A$ and choose $n$ such that $t \in A_{n}$. Since $\left\{\bar{A}_{i}: i<\omega\right\}$ is discrete, there is $s_{t}<_{T} t$ such that $\left[s_{t}, t\right]_{T} \cap \bigcup_{i \neq n} \bar{A}_{i}=\emptyset$. Let $\eta_{t}=\left[s_{t}, t\right)_{T}$ and $c_{t}(a)=n$ for each $a \in \eta_{t}$. This defines $\tilde{\eta}_{A}$ and $\tilde{c}_{\tilde{\eta}_{A}}$ as required. Let $f$ be a uniformizing function for $\tilde{c}_{\tilde{\eta}_{A}}$. For each $t \in A$ choose $r_{t}$ with $s_{t} \leq_{T} r_{t}<_{T} t$ such that $\forall a \in\left[r_{t}, t\right)_{T}\left(f(a)=c_{t}(a)\right)$. Then $\left\{A_{n} \cup \bigcup_{t \in A \cap A_{n}}\left(r_{t}, t\right)_{T}: n<\omega\right\}$ is a family of pairwise disjoint open subsets of $T$ which separate $\left\{A_{n}: n<\omega\right\}$. This proves the theorem.

The original version of Theorem 9 did not require in the hypothesis that $A$ is closed. This strengthening was pointed out by the referee to whom we are grateful.

If $\mathbb{T}$ is as in the previous theorem, then $\mathbb{T}$ is also normal, hence countably paracompact, by a result of Hanazawa [6]. The next section presents a dual result.

\section{$\S 3$. WEAK DIAMONDS}

For each $2 \leq n \leq \omega$ let $\Phi_{n}$ denote the following assertion:

$\forall F: \omega^{<\omega_{1}} \rightarrow n \exists g: \omega_{1} \rightarrow n \forall f: \omega_{1} \rightarrow \omega\left(\left\{\alpha<\omega_{1}: F(f\lceil\alpha)=g(\alpha)\}\right.\right.$ is stationary $)$.

It is easily seen that for $2 \leq m \leq n \leq \omega, \diamond \rightarrow \Phi_{n} \rightarrow \Phi_{m}$. Shelah [7] proved that the implications in the reversed directions do not hold and later showed that for $3 \leq n \leq \omega, \neg \Phi_{n}$ is consistent with GCH. We now state some properties of $\Phi_{\omega}$ (see [1] for more details) which are needed in the proof of Theorem 9 below.

Recall that $\mathcal{I}$ is the ideal of nonstationary subsets of $\omega_{1}$. It is well known that $\mathcal{I}$ is normal, i.e. if $\left\{I_{\nu}: \nu<\omega_{1}\right\} \subseteq \mathcal{I}$, then $\left\{\alpha<\omega_{1}: \exists \nu<\alpha\left(\alpha \in I_{\nu}\right)\right\} \in \mathcal{I}$. And in particular, $\mathcal{I}$ is countably complete, i.e. if $\left\{I_{\nu}: \nu<\omega\right\} \subseteq \mathcal{I}$, then $\bigcup_{\nu<\omega} I_{\nu} \in \mathcal{I}$. We say that $S \subseteq \omega_{1}$ is small if

$$
\exists F: \omega^{<\omega_{1}} \rightarrow \omega \forall g: \omega_{1} \rightarrow \omega \exists f: \omega_{1} \rightarrow \omega(\{\alpha \in S: F(f\lceil\alpha)=g(\alpha)\} \in \mathcal{I}) .
$$

Let $\mathcal{S}$ be a collection of all small subsets of $\omega_{1}$. Clearly, $\Phi_{\omega} \leftrightarrow \omega_{1} \notin \mathcal{S}$. For the next theorem we recall a result from $[1$.

Lemma 10. $\mathcal{S}$ is a normal ideal on $\omega_{1}$.

Theorem 11. If $\Phi_{\omega}$ holds, then no special Aronszajn tree is countably paracompact. 
Proof. Let $\mathbb{T}=\left\langle T, \leq_{T}\right\rangle$ be a special Aronszajn tree. By way of contradiction assume $\mathbb{T}$ is countably paracompact. We may assume that $T=\omega_{1}, \operatorname{ht}(\alpha, \mathbb{T})=\alpha$ for each limit $\alpha, \alpha \leq_{T} \beta \rightarrow \alpha \leq \beta$, and $\operatorname{ht}(\alpha, \mathbb{T})<\operatorname{ht}(\beta, \mathbb{T})$ for each $\alpha<\beta$ with limit $\beta$.

Let $A_{n}(n<\omega)$ be pairwise disjoint anti-chains of $\mathbb{T}$ with $T=\bigcup_{n<\omega} A_{n}$. Since $\mathcal{S}$ is countably complete and $\omega_{1} \notin \mathcal{S}$, there is an $m$ such that $A_{m} \notin \mathcal{S}$. Let $E=\left\{\alpha \in A_{m}:\right.$ limit $\left.\alpha\right\}$ and note that $E \notin \mathcal{S}$.

We define $F: \omega^{<\omega_{1}} \rightarrow \omega$ as follows: If $\alpha$ is a limit ordinal and $f \in \omega^{\alpha}$ is bounded on $(\beta, \alpha)_{T}$ for some $\beta<_{T} \alpha$, let $n$ be the largest value in the range of $f \uparrow(\beta, \alpha)_{T}$ whose set of preimages is $\leq_{T}$-unbounded in $(\beta, \alpha)_{T}$. In this case let $F(f)=n$. Let $F(f)=0$ otherwise.

Since $E \notin \mathcal{S}$, there is a $g \in \omega^{\omega_{1}}$ such that for all $f \in \omega^{\omega_{1}},\{\alpha \in E: F(f \mid \alpha)=$ $g(\alpha)\}$ is stationary in $\omega_{1}$. Let $E_{n}=\{\alpha \in E: g(\alpha)=n\}$. Since $E$ is an anti-chain in $\mathbb{T}$, it is closed and discrete so that $\left\{U_{n}=(T \backslash E) \cup E_{n}: n<\omega\right\}$ is an open cover of $T$. By countable paracompactness, let $\left\{V_{n}: n<\omega\right\}$ be a locally finite open refinement of $\left\{U_{n}: n<\omega\right\}$ such that $E_{n} \subseteq V_{n} \subseteq U_{n}$.

We use the cover $\left\{V_{n}: n<\omega\right\}$ to define $f: \omega_{1} \rightarrow \omega$. Let $\alpha \in T$. If $\operatorname{ht}(\alpha, \mathbb{T})$ is a successor ordinal, then $\{\alpha\}$ is open in $T$ and by countable paracompactness only finitely many $V_{n}$ intersect $\{\alpha\}$. In this case let $f(\alpha)=\max \left\{n+1: \alpha \in V_{n}\right\}$. Now suppose ht $(\alpha, \mathbb{T})$ is a limit ordinal and $f(\beta)$ has been defined for each $\beta<_{T} \alpha$. By countable paracompactness again, there is $\beta<_{T} \alpha$ such that $(\beta, \alpha]_{T}$ intersects only finitely many $V_{n}$ and $f$ is bounded on $(\beta, \alpha)_{T}$. Let $f(\alpha)$ be the largest value in the range of $f \uparrow(\beta, \alpha)_{T}$ whose set of preimages is $\leq_{T}$-unbounded in $(\beta, \alpha)_{T}$. This defines $f$.

We make an observation: For any $n<\omega$ and any $\alpha \in E_{n}, n<f(\alpha)$.

And now since $\left\{\alpha \in E: F(f\lceil\alpha)=g(\alpha)\}\right.$ is stationary, $E=\bigcup_{i<\omega} E_{i}$ and $\mathcal{I}$ is countably complete, there is $n<\omega$ such that $K=\left\{\alpha \in E_{n}: F(f \uparrow \alpha)=g(\alpha)\right\}$ is stationary, hence nonempty. Let $\alpha \in K$. Then $F(f\lceil\alpha)=g(\alpha)$. On the other hand, by definition of $E_{n}, g(\alpha)=n$ and by the above observation, $f(\alpha)>n$ so that $f(\alpha)>g(\alpha)$. Furthermore, since $\operatorname{ht}(\alpha, \mathbb{T})=\alpha$ is a limit ordinal, by definition of $f$, there is $\beta<_{T} \alpha$ such that $f$ is bounded on $(\beta, \alpha)_{T}$ and $f(\alpha)$ is the largest value that $f$ assumes on the interval $(\beta, \alpha)_{T}$ whose set of preimages is $\leq_{T}$-unbounded in $(\beta, \alpha)_{T}$. So by the definition of $F, F(f\lceil\alpha)=f(\alpha)$ and, together with $f(\alpha)>g(\alpha)$, we get $F(f\lceil\alpha)>g(\alpha)$. But this contradicts $F(f\lceil\alpha)=g(\alpha)$ and the theorem is proved.

As a corollary of this theorem and Theorem 9 we get that if $\Phi_{\omega}$ holds, then the uniformization fails for special Aronszajn trees. In fact, it was shown in [3] that a weaker assumption than $\Phi_{\omega}$, namely $2^{\omega}<2^{\omega_{1}}$, implies that normality fails in special Aronszajn trees, hence by Theorem 9, the uniformization also fails for such trees.

\section{REFERENCES}

[1] K. Devlin and S. Shelah, A weak version of $\diamond$ which follows from $2^{\omega}<2^{\omega_{1}}$, Israel Journal of Mathematics, Vol. 29, Nos. 2-3 (1978) 239-245. MR 57:9537

[2] K. Devlin and S. Shelah, Suslin properties and tree topologies, Proceedings of The London Mathematical Society, 39 (1979) 237-252. MR 80m:54031

[3] K. Devlin and S. Shelah, A note on the normal Moore space conjecture, Canadian Journal of Mathematics, 31 (1979) 241-252. MR 81d:54022 
[4] W. Fleissner, When is a Jone's space normal, Proceedings of the American Mathematical Society, 50 (1975) 375-378. MR 52:15384

[5] W. Fleissner, Remarks on Suslin Properties and Tree Topologies, Proceedings of the American Mathematical Society, 80 (1980) 320-326. MR 81k:54054

[6] M. Hanazawa, Note on Countable Paracompactness of Collection-wise Hausdorff tree topologies, Saitama Mathematics Journal, Vol. 2 (1984) 7-20.

[7] S. Shelah, Whitehead groups may not be free even assuming CH, II, Israel Journal of Mathematics, Vol. 35, No. 4 (1980) 257-285. MR 82h:03055

[8] S. Watson, Separation in countably metacompact spaces, Transactions of the American Mathematical Society, 290 (1985) 831-842. MR 87b:54016

Department of Mathematics, Massachusetts Institute of Technology, Cambridge, MASSACHUSETtS 02139 